\title{
Renal Heparan Sulfate Proteoglycans Modulate Fibroblast Growth Factor 2 Signaling in Experimental Chronic Transplant Dysfunction
}

Kirankumar Katta, ${ }^{*}$ Miriam Boersema, ${ }^{\dagger}$ Saritha Adepu, ${ }^{*}$ Heleen Rienstra, ${ }^{\dagger}$ Johanna W.A.M. Celie, ${ }^{\S}$ Rik Mencke, Grietje Molema, ${ }^{\dagger}$ Harry van Goor, ${ }^{\dagger}$ Jo H.M. Berden, ${ }^{\top}$ Gerjan Navis, ${ }^{*}$ Jan-Luuk Hillebrands, ${ }^{\dagger}$ and Jacob van den Born*

From the Division of Nephrology, * Department of Internal Medicine, the Department of Pathology and Medical Biology, ${ }^{\dagger}$ and the Immunology Section, ${ }^{\ddagger}$ Department of Cell Biology, University Medical Center Groningen, University of Groningen, Groningen; the Department of Pathology, ${ }^{\S}$ Academic Medical Center, Amsterdam; and the Department of Nephrology, Radboud University Nijmegen Medical Center, Nijmegen, the Netherlands

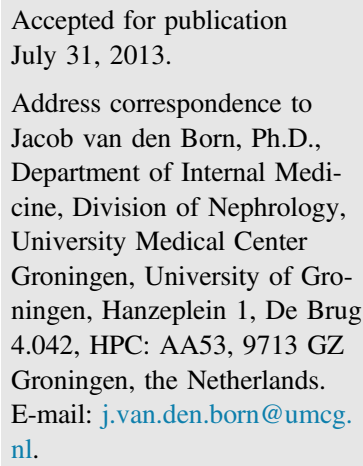

\begin{abstract}
Depending on the glycan structure, proteoglycans can act as coreceptors for growth factors. We hypothesized that proteoglycans and their growth factor ligands orchestrate tissue remodeling in chronic transplant dysfunction. We have previously shown perlecan to be selectively up-regulated in the glomeruli and arteries in a rat renal transplantation model. Using the same model, here we present quantitative RT-PCR profiling data on proteoglycans and growth factors from lasermicrodissected glomeruli, arterial tunicae mediae, and neointimae at 12 weeks after transplantation. In glomeruli and neointimae of allografts, selective induction of the matrix heparan sulfate proteoglycan perlecan was observed, along with massive accumulation of fibroblast growth factor 2 (FGF2). Profiling the heparan sulfate polysaccharide side chains revealed conversion from a non-FGF2-binding heparan sulfate phenotype in control and isografted kidneys toward a FGF2binding phenotype in allografts. In vitro experiments with perlecan-positive rat mesangial cells showed that FGF2-induced proliferation is dependent on sulfation and can be inhibited by exogenously added heparan sulfate. These findings indicate that matrix proteoglycans such as perlecan serve as functional docking platforms for FGF2 in chronic transplant dysfunction. We speculate that heparin-like glycomimetics could be a promising intervention to retard development of glomerulosclerosis and neointima formation in chronic transplant dysfunction. (Am J Pathol 2013, 183: 1571-1584; http://dx.doi.org/10.1016/j.ajpath.2013.07.030)
\end{abstract}

Renal chronic transplant dysfunction (CTD) is the leading cause of long-term loss of transplanted kidneys. ${ }^{1,2}$ CTD is the result of tissue remodeling in the intrarenal arteries, glomeruli, and tubulointerstitium that leads to transplant vasculopathy, focal glomerulosclerosis (FGS), interstitial fibrosis, and tubular atrophy. ${ }^{2-4}$ These lesions are characterized by accumulation of extracellular matrix, activation of mesangial cells, interstitial myofibroblasts, tubular epithelial cells, and chronic inflammation. To date, in the absence of knowledge of the pathogenetic mechanisms leading to development of these lesions, no effective therapies are available to prevent or treat renal CTD. Progressive loss can only be retarded by antihypertensive and antiproteinuric treatment in combination with lipid-lowering drugs.
Recently, our research group reported accumulation of the heparan sulfate proteoglycans (HSPGs) collagen type XVIII and perlecan during glomerular and vascular tissue remodeling in CTD in rats. ${ }^{5}$ However, whether and how these proteoglycans mediate tissue remodeling in CTD is as yet unknown. In native kidney diseases and ischemia-reperfusion injury, we have already demonstrated that HSPGs are involved in

Supported by the Dutch Kidney Foundation (C03.6015; M.B., H.R., and J.-L.H.) and the Graduate School of Medical Sciences of the University Medical Center Groningen (K.K.). Confocal microscopy and TissueFAXS analysis were performed at the University Medical Center Groningen Imaging Center, which is supported by the Netherlands Organization for Health Research and Development (ZonMW grant 40-00506-98-9021).

K.K. and M.B. contributed equally to this work. 
leukocyte influx and proteinuria-mediated renal injury. ${ }^{6-8}$ Perlecan is a large, modular, pericellular HSPG with a spatial distribution that enables control over major cellsignaling events such as migration, proliferation, and differentiation in response to bound growth factors. Perlecan is composed of five different domains, of which the C-terminal domain, named endorepellin, is best known for its antiangiogenic properties. ${ }^{9,10}$

The carbohydrate side chains (ie, the glycosaminoglycans) are attached to proteoglycan core proteins and can bind a variety of ligands, depending on their highly variable composition (mainly variations in patterns of $\mathrm{N}$ - and $\mathrm{O}$ sulfation). ${ }^{11}$ Along with chondroitin sulfate and dermatan sulfate proteoglycans, HSPGs form the large majority of the proteoglycan family. Potential ligands include chemokines ${ }^{11}$ and growth factors such as basic fibroblast growth factor (bFGF/FGF2). ${ }^{12,13}$ Proteoglycans are highly involved in morphogenesis and in tissue remodeling processes. ${ }^{14-19}$

Studies in nontransplantation models of renal and vascular disease have identified potential roles of FGF2 in FGS and neointima formation. ${ }^{20-24}$ These findings led us to hypothesize that interaction of FGF2 with proteoglycans also affects tissue remodeling processes in CTD. To test this hypothesis, we used an experimental rat CTD model. We microdissected glomeruli, the arterial media, and the neointima and performed low-density quantitative RT-PCR (RT-qPCR) analysis for matrix and cell-surface proteoglycans and FGF2. In addition, we profiled the heparan sulfate (HS) polysaccharide side chains by anti-HS monoclonal antibodies (mAbs) and their binding capacity for FGF2 and L-selectin. Functionally, we investigated the HSPG involvement of FGF2-driven mesangial proliferation. Our data indicate spatial proteoglycan involvement in CTD and thus suggest a potential target for intervention therapy in the future.

\section{Materials and Methods}

\section{Rats}

Inbred female Dark Agouti (DA) rats (175 to $210 \mathrm{~g}$ ) were obtained from Harlan Laboratories (Horst, the Netherlands; Indianapolis, IN) and inbred male Wistar Furth (WF) rats (240 to $295 \mathrm{~g}$ ) from Charles River Laboratories International (l'Arbresle, France; Wilmington, MA). All animals received care in compliance with the NIH Guide for the Care and Use of Laboratory Animals (revised 1985), the University of Groningen guidelines for animal husbandry, and the Dutch Law on Experimental Animal Care.

\section{Kidney Transplantation and Experimental Groups}

Female DA kidney allografts were orthotopically transplanted into male Wistar-Furth recipients, as described previously. ${ }^{5,25,26}$ Cold ischemic time ranged from 16 to 38 minutes; warm ischemic time ranged from 19 to 32 minutes.
Recipients received $5 \mathrm{mg} / \mathrm{kg}$ s.c. cyclosporin A (Sandimmune; Novartis, Basel, Switzerland) on the first 10 days after transplantation. The contralateral kidney was removed 8 to 14 days after transplantation. Total follow-up time was 12 weeks, unless animals had to be sacrificed sooner because of renal failure. Allografts that developed severe CTD were used in this study $(n=5)$. Further characteristics of the model are described elsewhere. ${ }^{5,25,26}$ Nontransplanted DA kidneys $(n=5)$ and DA-to-DA isografted kidneys $(n=5)$ served as controls.

\section{Laser Microdissection and Gene Expression Analysis}

Laser microdissection, RNA isolation, and RT-qPCR were performed essentially according to Asgeirsdottir et al. ${ }^{27}$ Glomeruli and various layers (including the tunica media and the neointima) of larger arteries were separately dissected from nine serial sections per kidney. An average of 194 (range, 71 to 308) glomeruli and 63 (range, 25 to 117) arteries were dissected from each kidney. Glomeruli and the various arterial layers were isolated from allografted, isografted, and nontransplanted kidneys ( $n=5$ per group).

Total RNA was also isolated from whole-kidney sections from allograft, isograft, and nontransplanted animals $(n=3$ per group). Total RNA was isolated from microdissected structures and whole kidney using an RNeasy micro kit (Qiagen, Hilden, Germany; Valencia, CA). Reverse transcription was performed using Invitrogen SuperScript III Reverse Transcriptase (Life Technologies, Breda, the Netherlands; Carlsbad, CA) and random hexamer primers (Promega, Leiden, the Netherlands; Madison, WI). Gene expression was analyzed with a custom-made microfluidic card-based low-density array (Life Technologies-Applied Biosystems, Nieuwerkerk a/d IJssel, the Netherlands) using an ABI Prism 7900HT sequence detection system (Life Technologies-Applied Biosystems).

Relative mRNA levels were calculated as $2^{-\Delta \mathrm{CT}}$, where $\Delta \mathrm{C}_{\mathrm{T}}=\mathrm{C}_{\mathrm{T}(\text { gene of interest })}-\mathrm{C}_{\mathrm{T}(\beta \text {-actin) }} . \mathrm{C}_{\mathrm{T}}$ values that were beyond detection level were set manually to 50 . Composition of the low-density array is presented in Table 1.

\section{Single and Double Immunofluorescence for Proteoglycans and FGF2 on Rat Kidney Sections}

Frozen sections ( $4 \mu \mathrm{m}$ thick) were fixed in acetone or $4 \%$ formaldehyde and were blocked for endogenous peroxidase activity with $0.03 \% \mathrm{H}_{2} \mathrm{O}_{2}$ if appropriate. Sections were blocked with normal goat or rabbit serum. Sections were incubated for 1 hour with the following primary antibodies: mouse anti-human FGF2 (PeproTech, London, UK; Rocky Hill, NJ), mouse anti-HS mAb JM-403, ${ }^{28}$ mouse anti-HS stub mAb 3 G10 (Seikagaku, Tokyo, Japan), ${ }^{29}$ and mouse anti-rat perlecan (clone 10B2; kindly provided by J.R. Couchman, University of Copenhagen). Binding of primary antibodies was detected by incubating the sections for 30 minutes with secondary antibodies diluted in PBS with 5\% 
Table 1 Composition of Low-Density Array

\begin{tabular}{|c|c|c|c|}
\hline Gene name & $\begin{array}{l}\text { Protein } \\
\text { short } \\
\text { name }\end{array}$ & $\begin{array}{l}\text { Gene } \\
\text { symbol }\end{array}$ & Assay ID \\
\hline \multicolumn{4}{|l|}{ Matrix molecules } \\
\hline $\begin{array}{l}\text { Collagen, type I, } \\
\text { alpha } 1\end{array}$ & Collagen I & Col1a1 & Rn01463848_m1 \\
\hline $\begin{array}{l}\text { Collagen, type IV, } \\
\text { alpha } 1\end{array}$ & Collagen IV & Col4a1 & Rn01482925_m1 \\
\hline \multicolumn{4}{|l|}{ Proteoglycans } \\
\hline Perlecan & Perlecan & LOC313641 & Rn01515780_g1 \\
\hline Agrin & Agrin & Agrn & Rn00598349_m1 \\
\hline Versican & Versican & Vcan & Rn01493755_m1 \\
\hline Biglycan & Biglycan & $B g n$ & Rn00567229_m1 \\
\hline Syndecan 1 & Syndecan-1 & Sdc1 & Rn00564662_m1 \\
\hline Syndecan 4 & Syndecan-4 & Sdc4 & Rn00561900_m1 \\
\hline \multicolumn{4}{|l|}{ Growth factors } \\
\hline $\begin{array}{l}\text { Fibroblast growth } \\
\text { factor } 2\end{array}$ & FGF2 & Fgf2 & Rn00570809_m1 \\
\hline $\begin{array}{l}\text { Transforming growth } \\
\text { factor, beta } 1\end{array}$ & TGF- $\beta 1$ & $\operatorname{Tgfb1}$ & Rn01475963_m1 \\
\hline \multicolumn{4}{|l|}{ Reference genes } \\
\hline Beta actin & $\beta$-Actin & Actb & Rn00667869_m1 \\
\hline Beta 2 microglobulin & $\mathrm{B} 2 \mathrm{M}$ & $B 2 m$ & Rn00560865_m1 \\
\hline Eukaryotic $18 \mathrm{~S}$ rRNA & $18 \mathrm{~S}$ & 185 & Hs99999901_s1 \\
\hline
\end{tabular}

normal rat serum: goat anti-mouse $\mathrm{IgG}_{1}$ horseradish peroxidase (HRP) (SouthernBiotech, Birmingham, AL), rabbit anti-mouse IgM HRP (Dako, Heverlee, Belgium; Carpinteria, CA), or goat anti-mouse $\operatorname{IgG}_{1}$ Alexa Fluor 488 (Life Technologies). HRP activity was visualized using a tyramide signal amplification tetramethylrhodamine system (TSA; PerkinElmer, Waltham, MA). All fluorescence microscopy was performed using a Leica DMLB microscope (Leica Microsystems, Rijswijk, the Netherlands; Wetzlar, Germany) equipped with a Leica DC300F camera and Leica QWin software version 2.8 .

\section{Detection of Proliferating Mesangial Cells Using Ki-67 in Rat Kidney Sections}

Frozen sections (4 $\mu \mathrm{m}$ thick) were fixed in $2 \%$ paraformaldehyde for 10 minutes and then were permeabilized in $0.5 \%$ Triton X-100 for 5 minutes. Sections were incubated for 1 hour with mouse anti-rat perlecan $\left(\mathrm{mIgG}_{1}\right.$, clone 10B2) or a cocktail of mouse anti-rat CD90 (alias Thy-1) $\left(\mathrm{mIgG}_{1}\right.$, clone $\mathrm{OX7}$, tissue culture supernatant) and polyclonal rabbit anti-Ki-67 (Novocastra NCL-Ki67p; Leica Biosystems, Wetzlar, Germany) antibodies. After primary antibody incubation, sections were blocked for endogenous peroxidase activity with $0.03 \% \mathrm{H}_{2} \mathrm{O}_{2}$ if appropriate. Binding of primary antibodies was detected by incubating the sections for 30 minutes with appropriate secondary antibodies diluted in PBS-1\% bovine serum albumin with 3\% normal rat serum. The secondary antibodies used were goat anti-mouse $\mathrm{IgG}_{1}$ Alexa Fluor 488 (Life Technologies) and goat anti-rabbit IgG HRP (Dako). HRP activity was visualized using the TSA tetramethylrhodamine system (PerkinElmer). Nuclei were counterstained with DAPI. Slides were mounted with Aqua PolyMount medium (Polysciences, Warrington, PA). Images were acquired with an Axio Observer Z1 inverted microscope (Carl Zeiss Microscopy, Jena, Germany) equipped with TissueFAXS acquisition software version 2.0.4 (TissueGnostics, Vienna, Austria; Tarzana, CA).

\section{Ligand Binding Assays on Rat Kidney Sections}

To detect capacity of renal proteoglycans to bind FGF2, formalin-fixed rat renal sections were incubated with $1 \mu \mathrm{g} /$ $\mathrm{mL}$ recombinant human FGF2 (recFGF2; PeproTech) for 60 minutes. After a washing step, staining was continued according to the FGF2 staining protocol as described above. Use of formalin fixation essentially avoids recognition of endogenous renal FGF2 by anti-FGF2 antibodies. Similarly, paraformaldehyde-fixed rat renal sections were incubated with $1 \mu \mathrm{g} / \mathrm{mL}$ L-selectin- $\mathrm{Fc}$ recombinant chimeric protein and visualized as described previously. ${ }^{30}$ To confirm that the observed binding pattern was mediated by HS proteoglycans, the sections were pretreated with $0.05 \mathrm{U} / \mathrm{mL}$ heparitinase I (EC 4.2.2.8, Flavobacterium heparinum; Seikagaku) for 1 hour at $37^{\circ} \mathrm{C}$ in a humidified chamber.

\section{FGF2 Binding Assays in ELISA}

In an enzyme-linked immunosorbent assay (ELISA) approach, we evaluated the capability of fluid phase HS-like polysaccharides to compete for the interaction of recFGF2 with immobilized perlecan. To this end, MaxiSorp 96-well plates (Nalge Nunc International, Rochester, NY) were coated overnight in PBS with $5 \mu \mathrm{g} / \mathrm{mL}$ perlecan (SigmaAldrich, St. Louis, MO). After a washing in PBS with $0.05 \%$ Tween 20, wells were blocked with 5\% nonfat milk powder in Tris-buffered saline for 1 hour. In a separate microtiter plate, $0.5 \mu \mathrm{g} / \mathrm{mL}$ recFGF2 was incubated for 30 minutes with a dilution range of different HS-like polysaccharides and then was transferred to the ELISA plate after the wells had been washed again. Incubation in the wells took 1 hour. The wells were washed again, and monoclonal mouse anti-FGF2 antibodies were added to the wells $(0.5 \mu \mathrm{g} / \mathrm{mL}$ Tris-buffered saline-Tween 20). Secondary antibody was added after a washing step (HRP-labeled rabbit anti-mouse IgG, 1:5000; Dako). Secondary antibody was detected with $3,3^{\prime}, 5,5^{\prime}$-tetramethylbenzidine substrate (Sigma-Aldrich) for 15 minutes in the dark, and the reaction was stopped by adding $1.5 \mathrm{~N}$ $\mathrm{H}_{2} \mathrm{SO}_{4}$. Absorbance was measured at $450 \mathrm{~nm}$ in a microplate reader. All incubations were performed in a volume of 100 $\mu \mathrm{L}$ per well at room temperature. Polysaccharides used in the FGF2 competition ELISA were heparin from porcine intestinal mucosa (Sigma-Aldrich) and HS from bovine kidney (Seikagaku); N-sulfation of HS from bovine kidney and Nand O-desulfation of heparin from porcine intestine were performed as described before. ${ }^{8}$ HS from human aorta was 
isolated essentially according to the method of Iverius. ${ }^{31} \mathrm{HS}$ from Engelbreth-Holm-Swarm murine sarcoma was obtained from Seikagaku.

To evaluate FGF2 binding by perlecan from renal lysates, a slightly modified ELISA approach was followed. Perlecan from renal lysates $(10 \mu \mathrm{g}$ protein $/ \mathrm{mL}$ carbonate buffer) was immunocaptured on monoclonal mouse anti-rat perlecan antibody (1:1000 in PBS; mAb 10B2; provided by J.R. Couchman) immobilized in MaxiSorp 96-well plates. After appropriate blocking, captured renal perlecan was incubated with $0.5 \mu \mathrm{g} / \mathrm{mL}$ recFGF2, followed by biotinylated antiFGF2 mAb (clone JKFb-2, $1 \mu \mathrm{g} / \mathrm{mL}$ Tris-buffered salineTween 20; Novus Biologicals, Cambridge, UK) and then by HRP-conjugated streptavidin (1:5000 in Tris-buffered saline-Tween 20). Substrate reaction and plate reading was as described above.

\section{FGF2 Stimulation Assay on Rat Mesangial Cells}

Rat mesangial cells (positive for Thy 1.1, perlecan, and $\alpha$-smooth muscle actin; passage 11 to 15 ) were cultured in 24-well plates in Dulbecco's modified Eagle's medium supplemented with $25 \mathrm{mmol} / \mathrm{L}$ HEPES, $4.5 \mathrm{mg} / \mathrm{mL}$ glucose, pyridoxine, $1 \mathrm{mmol} / \mathrm{L}$ pyruvate, $50 \mathrm{ng} / \mathrm{mL}$ insulin, and $10 \%$ fetal bovine serum. Before stimulation with FGF2, cells were grown until confluency, serum-deprived $(0.5 \%$ serum $)$ for 24 hours, and then incubated with 0.031 to $8 \mathrm{ng} / \mathrm{mL}$ of FGF2 for 24 hours. For measurement of proliferation, 0.5 $\mu \mathrm{Ci} / \mathrm{mL}\left[{ }^{3} \mathrm{H}\right]$ thymidine (GE Healthcare) was added to the cultures. After 24 hours, 5\% trichloroacetic acid precipitable material was dissolved in $0.1 \%$ SDS, OptiPhase HiSafe 3 liquid scintillation cocktail (PerkinElmer) was added, and radioactivity was counted in a Wallac 1214 Rackbeta liquid scintillation counter (PerkinElmer). To study whether FGF2-induced proliferation of mesangial cells was reduced in the presence of exogenous HS, mesangial cells were stimulated with $0.5 \mathrm{ng} / \mathrm{mL}$ FGF2 for 24 hours in the presence of various concentrations $(0,8,32$, and $128 \mu \mathrm{g} / \mathrm{mL})$ of exogenous HS from bovine kidney (HSBK; Seikagaku). Proliferation was measured as described above.

To study whether proteoglycan sulfation was reduced by chlorate, mesangial cells were cultured for 24 hours in the presence of 5 to $25 \mathrm{mmol} / \mathrm{L}$ sodium chlorate (SigmaAldrich) and $2 \mu \mathrm{Ci} / \mathrm{mL}\left[{ }^{35} \mathrm{~S}\right]$ sulfate (GE Healthcare, Little Chalfont, UK). Incorporation of $\left[{ }^{35} \mathrm{~S}\right]$ sulfate into proteoglycans was quantified as described above for $\left[{ }^{3} \mathrm{H}\right]$ thymidine incorporation. Finally, to analyze whether chlorate impairs FGF2-induced proliferation of mesangial cells, cells

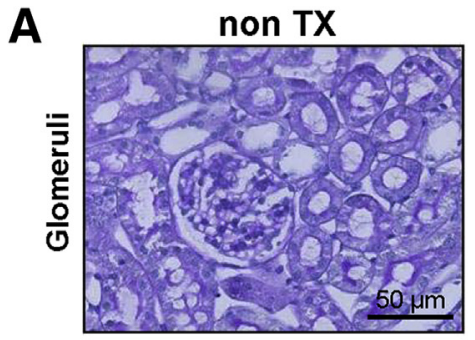

B

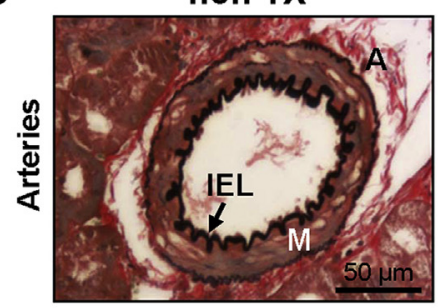

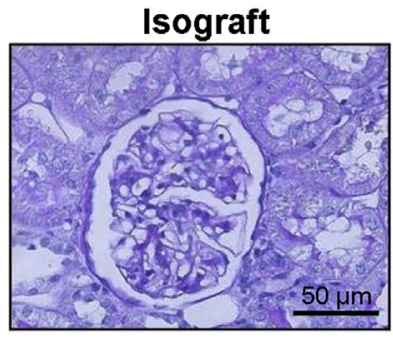

Isograft

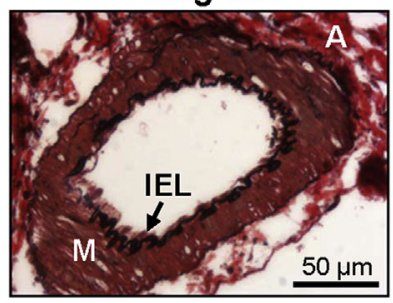

Allograft

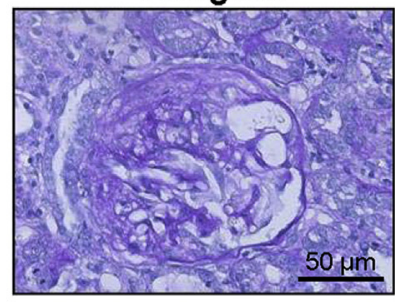

Allograft

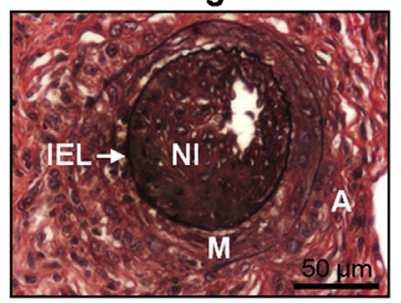

C

Col IVa1
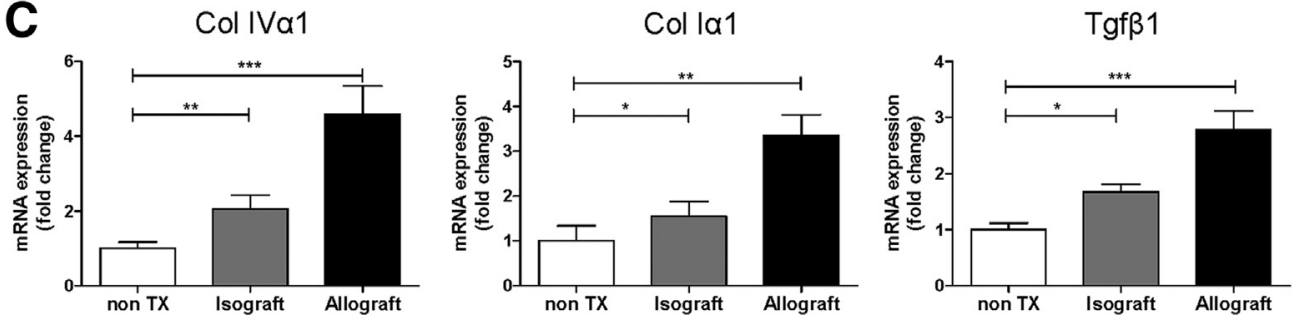

Figure 1 Chronic transplant dysfunction in renal allografts. A: Representative photomicrographs showing development of glomerulosclerosis in allografted kidneys, in contrast to isografted and control kidneys (PAS staining). B: Neointima formation was observed in allografts, but not in isografts or controls (Verhoeff staining). C: RT-qPCR analysis was performed on RNA isolated from microdissected glomeruli from nontransplanted control tissue, isografts, and allografts. Expression levels of ColIV $\alpha 1$, ColI $\alpha 1$, and TGF- $\beta 1$ are expressed as fold change relative to nontransplanted control kidneys. Data are expressed as means \pm SEM. $n=5$ per group. ${ }^{*} P<0.05,{ }^{* *} P<0.01$, and ${ }^{* *} * P<0.001$. Scale bar $=50 \mu \mathrm{m}$. A, adventitia; IEL, internal elastic lamina; M, media; NI, neointima; TX, transplanted. 
were stimulated with $0.5 \mathrm{ng} / \mathrm{mL}$ FGF2 for fixed times $(0$, $0.5,1,2,4$, and 24 hours) in the presence or absence of 25 $\mathrm{mmol} / \mathrm{L}$ sodium chlorate and in the presence of $0.5 \mu \mathrm{Ci} / \mathrm{mL}$ $\left[{ }^{3} \mathrm{H}\right]$ thymidine. Total culture time was 24 hours. Proliferation was determined as described above. Before stimulation with FGF2, cells were grown until confluency, serumdeprived $(0.5 \%$ serum $)$ for 24 hours, and incubated with FGF2 for 24 hours. Proliferation was measured by adding $0.5 \mu \mathrm{Ci} / \mathrm{mL}\left[{ }^{3} \mathrm{H}\right]$ thymidine (GE Healthcare) for 24 hours to the cultures. After 24 hours, 5\% trichloroacetic acid precipitable material was dissolved in $0.1 \%$ SDS, OptiPhase HiSafe 3 cocktail (PerkinElmer) was added, and radioactivity was counted in a Wallac 1214 Rackbeta liquid scintillation counter (PerkinElmer). Incorporation of $\left[{ }^{35} \mathrm{~S}\right]$ sulfate into proteoglycans was quantified similarly as described above for $\left[{ }^{3} \mathrm{H}\right]$ thymidine incorporation.

\section{Detection of Perlecan Expression on Proliferating Rat Mesangial Cells}

Rat mesangial cells were cultured as described above. After expansion, cells were seeded on coverslips and serumstarved for 24 hours in medium containing $0.5 \%$ fetal calf serum (FCS) after attachment. Subsequently, cells were stimulated for 48 hours in medium containing either $2 \%$ or $10 \%$ FCS. After stimulation, cells were fixed in $2 \%$ paraformaldehyde and double-stained for $\alpha$-SMA ( $\mathrm{mIgG}_{2 \mathrm{a}}$, clone $1 \mathrm{~A} 4$; Dako) and perlecan $\left(\mathrm{mIgG}_{1}\right.$, clone $\left.10 \mathrm{~B} 2\right)$ as described above. Binding of primary antibodies was detected by incubating the sections for 30 minutes with goat antimouse $\mathrm{IgG}_{1}$ Alexa Fluor 488 and goat anti-mouse $\mathrm{IgG}_{2 \mathrm{a}}$ Alexa Fluor 555 (Life Technologies). Nuclei were counterstained with DAPI. Slides were mounted with Aqua PolyMount medium (Polysciences). Confocal microscopy was performed using an inverted microscope (Zeiss LSM 780 NLO; Axio Observer Z1). To quantify total cell numbers and numbers of perlecan expressing mesangial cells, coverslips were scanned using TissueFAXS acquisition software (TissueGostics) on a Zeiss Axio Observer Z1 inverted microscope. Quantitative analyses were performed using TissueQuest fluorescence analysis software (TissueGnostics).

\section{Statistical Analysis}

mRNA expression levels were analyzed using a one-way analysis of variance with Tukey's post hoc test. $P$ values of $<0.05$ were considered statistically significant (IBM SPSS software version 18; IBM, Armonk, NY). Statistical outliers, as detected by Grubbs' test for outliers, were excluded from analyses. Mesangial cell culture data were expressed as means \pm SEM and analyzed by one-way analysis of variance. If overall $P<0.05$, Bonferroni's
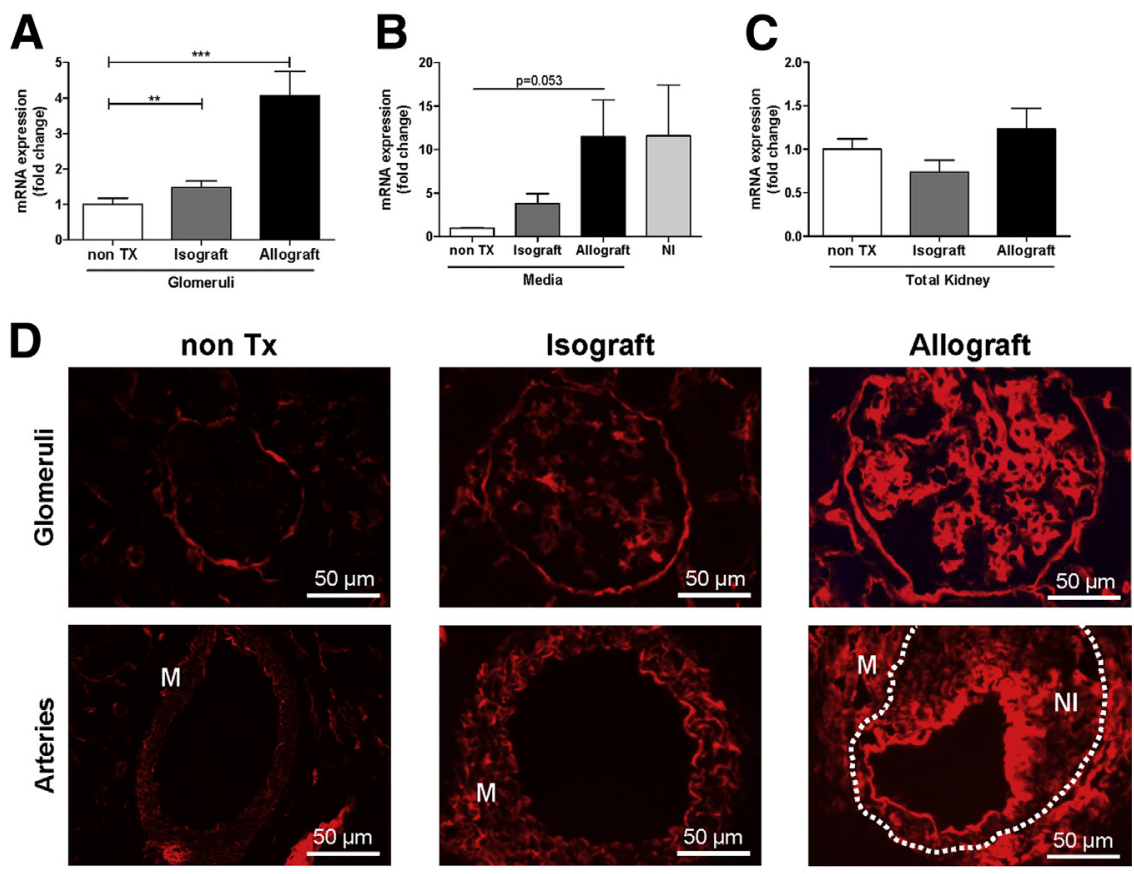

\section{$\mathbf{E}$}

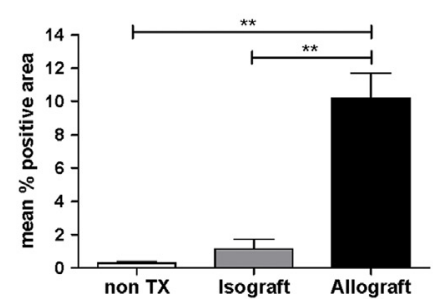

Figure 2 Up-regulation of perlecan in glomeruli and neointimae of renal allografts with CTD. A-C: Perlecan expression measured by RTqPCR in glomeruli (A), arterial mediae (B), and whole-kidney tissue (C). Expression levels of perlecan are expressed as fold change relative to nontransplanted control kidneys. D: Immunofluorescence demonstrates perlecan accumulation in glomeruli and neointimae of allografts with CTD. In isografted kidneys, a minor increase of perlecan is seen in glomeruli and arterial mediae, compared with control tissue. The internal elastic lamina is marked by a dotted line. E: Quantification of glomerular perlecan expression by digital image analysis revealed a significant increase in allografted kidneys. Data are expressed as means \pm SEM. $n=5$ per group (A and $\mathbf{B}$ ); $n=3$ per group (C). ${ }^{* *} P<0.01,{ }^{* * *} P<0.001$. Scale bar $=50$ $\mu \mathrm{m}$. 
Table 2 Basic Characteristics of the HS Epitopes Recognized by the Two Anti-HS mAbs, FGF2, and L-selectin

\begin{tabular}{ll}
\hline Anti-HS mAb or HS-binding protein & Basic characteristics of the HS epitope \\
\hline Anti-HS mAb JM-403 & GlcUA-rich sequences with N-unsubstituted GlcN units in low sulfated HS \\
Anti-HS mAb 3G10 & Desaturated uronate residues after digestion with heparitinase (GlcNAc/NS $\alpha 1-4$ GlcA linkage ${ }^{29}$ ) \\
FGF2 & $2-0$-sulfation in heparin and HS S2 $^{32}$ \\
L-selectin & $6-0$-sulfated GlcN units in HS and heparin ${ }^{33}$ \\
\hline
\end{tabular}

multiple comparison test was performed (GraphPad Prism version 5.0; GraphPad Software, San Diego, CA).

\section{Results}

\section{Development of Chronic Transplant Dysfunction in Renal Allografts}

Progressive renal function loss was evidenced in the allografted kidneys by an approximately $50 \%$ loss in creatinine clearance and an approximately 15 -fold increase in urinary protein excretion at 8 weeks after transplantation, neither of which was observed in isografted kidneys, as we have previously shown in this model. ${ }^{5,25,26}$ Allografted rats developed hypertension and showed development of severe CTD with FGS (Figure 1A) and arterial neointima formation (Figure 1B). In isografts, development of FGS was minimal and no neointima formation was observed. $5,25,26$ The development of FGS coincided with significantly increased expression of the profibrotic factors collagen type I (ColI $\alpha 1)$, collagen type IV (ColIV $\alpha 1$ ), and transforming growth factor $\beta 1$ (TGF- $\beta 1$ ), as determined by RT-qPCR on microdissected glomeruli (Figure 1C). These molecular responses were also observed in RNA isolated from microdissected arterial medial and neointimal tissue, as well as in whole-kidney material. ${ }^{26}$ These findings confirm development of CTD in our rat transplantation model.

\section{Induction of Glomerular and Neointimal Perlecan Expression in CTD}

Proteoglycans can modulate growth factor responses by virtue of their glycosaminoglycan side chains. We used RTqPCR to profile the matrix HS proteoglycans agrin and

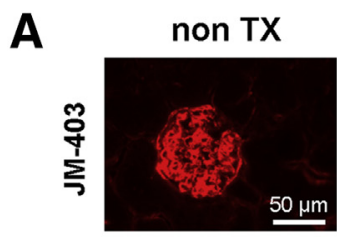

B
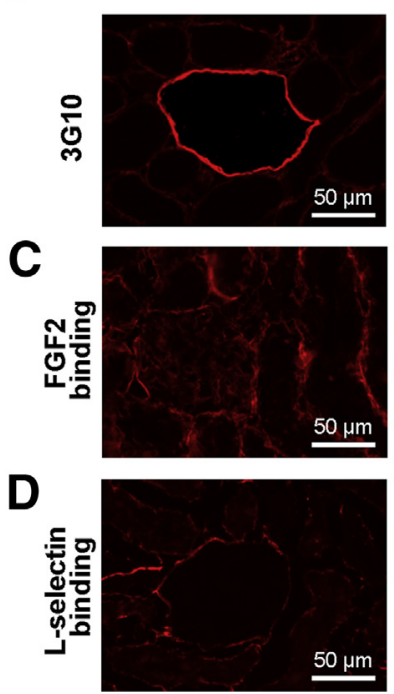

Isograft

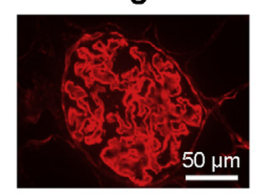

Allograft

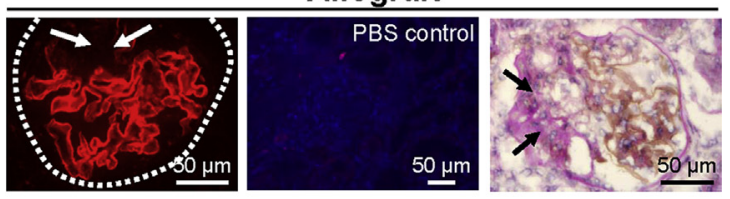

Allograft
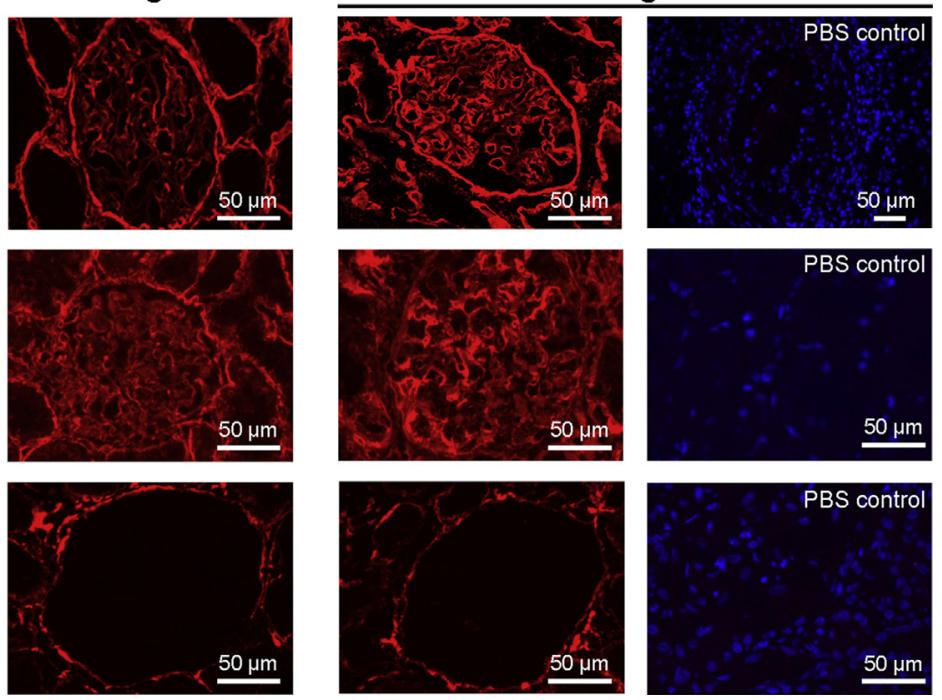

Figure 3 Modification of glomerular HS structure in allografts with CTD. Glomerular expression of HS was evaluated by two anti-HS mAbs, JM-403 (A) and $3 \mathrm{G} 10$ (B), as well as by binding of FGF2 (C) and L-selectin (D). In allografts, glomerular JM-403 staining (A) is partly lost in sclerotic areas (arrows), as evidenced by loss of JM-403 staining (brown) in PAS-positive sclerotic area. In contrast, HS expression as evidenced by 3 G10 (B) and FGF2 binding capacity (C) is increased in glomeruli in allografts. L-selectin (D) does not bind to glomerular HS of control, isografted, or allografted kidneys, although HS binds L-selectin in Bowman's capsule and in tubular basement membranes. Blanks (PBS control) show staining of allograft renal tissue without anti-HS mAb (A and B) or ligand binding assay without incubation with FGF2 (C) or L-selectin (D); double-staining with DAPI (blue) is used to visualize the nuclei of renal material. Bowman's capsule is marked by a dotted line (A). Scale bar $=50 \mu \mathrm{m}$. 
perlecan, the matrix chondroitin sulfate and dermatan sulfate proteoglycans versican and biglycan, and the major epithelial cell-surface proteoglycans syndecan-1 and -4 . The PCR analysis showed the expression of the basement membrane HSPG perlecan to be up-regulated mainly in the glomeruli (Figure 2A) and the mediae and neointimae (Figure 2B) of the allografts. However, the localized upregulation of perlecan revealed by RT-qPCR in glomeruli, mediae, and neointimae was not observed with RT-qPCR analysis performed on whole-kidney RNA isolates (Figure 2C), which points to the need for using microdissection of specific structures to demonstrate differential proteoglycan expression in specific renal compartments.

Use of immunofluorescence revealed that expression of perlecan was virtually absent in the glomerular basement membranes of nontransplanted control kidneys. In the glomerular basement membranes of isografts, perlecan expression was increased, compared with control kidneys, but to a far lesser extent than the increase observed in allografts (Figure 2D). In glomeruli with FGS in allografts, strong expression of perlecan was observed in the glomerular basement membranes and mesangial areas (Figure 2D). In control tissue isografts and allografts, Bowman's capsule was positive for perlecan. Immunofluorescence also revealed perlecan expression in the mediae and in the newly formed neointimae present in allografts (Figure 2D). Quantification of glomerular perlecan expression by digital image analysis (ImageJ version 1.48b; NIH, Bethesda, MD) revealed a significant increase in perlecan expression in the glomeruli of allografted kidneys, compared with isografts $(P$ $<0.01)$ and nontransplanted kidneys $(P<0.01)$ (Figure 2E).

No significant differences in expression levels were found for syndecan-1 and -4 , agrin, and biglycan, although these proteoglycans were expressed in the neointimae based on RT-qPCR data (Supplemental Figure S1). Versican expression was significantly up-regulated in the media of the allografted kidneys (Supplemental Figure S1E), which consistent with a promigratory phenotype of the vascular smooth muscle cells. This proteoglycan profiling led us to conclude that glomerular and neointimal perlecan is upregulated in renal allografts at both the mRNA and the protein level.

\section{HS Profiling}

Because HS proteoglycans are involved in morphogenesis and tissue remodeling mainly by their HS glycosaminoglycan side chains, we profiled HS polysaccharide structures in renal sections. To this end, we visualized HS epitopes with anti-HS mAbs JM-403 and 3G10 and with a tissue ligand binding assay using the HS-binding growth factor FGF2 and HS-binding adhesion molecule L-selectin. Characteristics of the corresponding HS epitopes are presented in Table 2.

Anti-HS mAb JM-403 showed clear staining of the glomerular basement membranes in control and isografted kidneys (Figure 3A). Mesangial staining was weak for $\mathrm{mAb}$
JM-403. In allografts, however, glomerular JM-403 HS staining was partly or completely lost in sclerotic areas (Figure 3A), likely because of increased HS sulfation. PASpositive sclerotic tissue was negative for anti-HS mAb JM403. The 3G10 HS epitope, which becomes available after
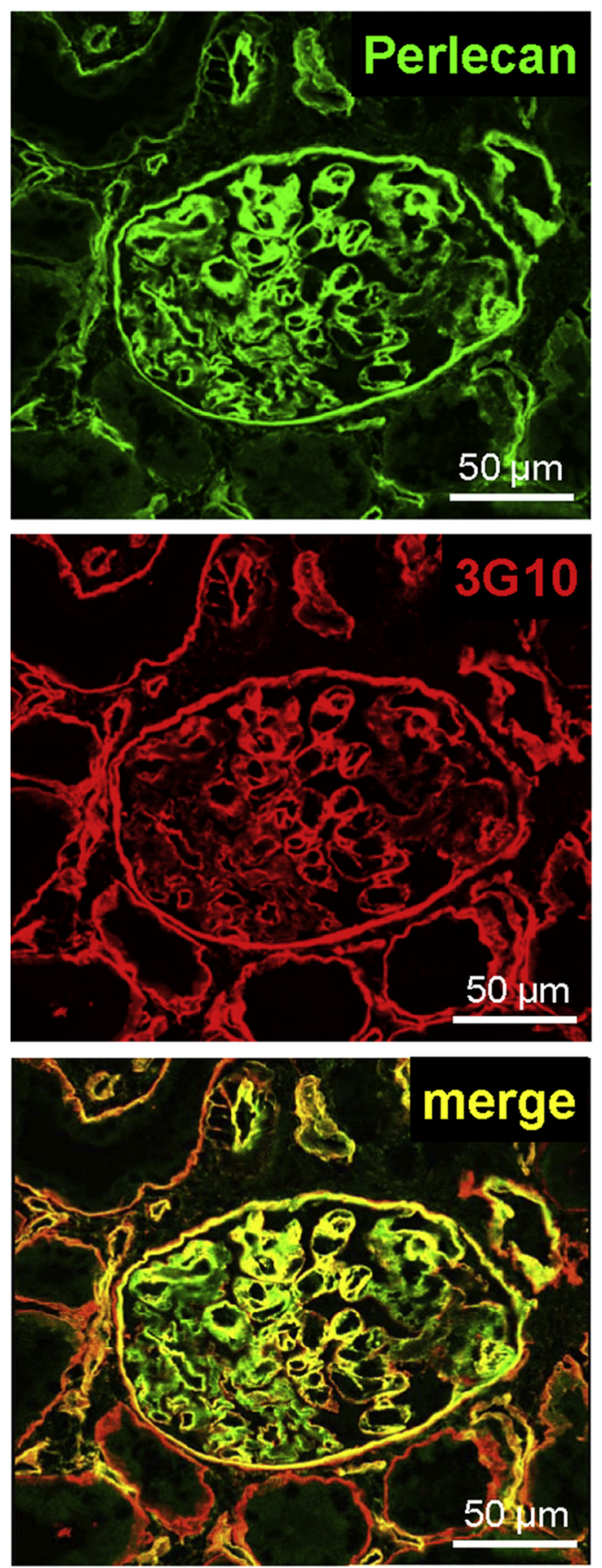

Figure 4 Perlecan and HS double-staining in glomeruli of allografted kidneys. In a double-immunofluorescent staining protocol, perlecan and HS (mAb 3G10) are seen to stain identical structures in the glomerulus (namely, Bowman's capsule, the thickened glomerular basement membranes, and enlarged mesangial matrix). mAb $3 \mathrm{G} 10$ also stains some tubular basement membranes that are barely positive for perlecan and most likely recognize HS bound to agrin and/or collagen type XVIII. Scale bar $=50 \mu \mathrm{m}$. 

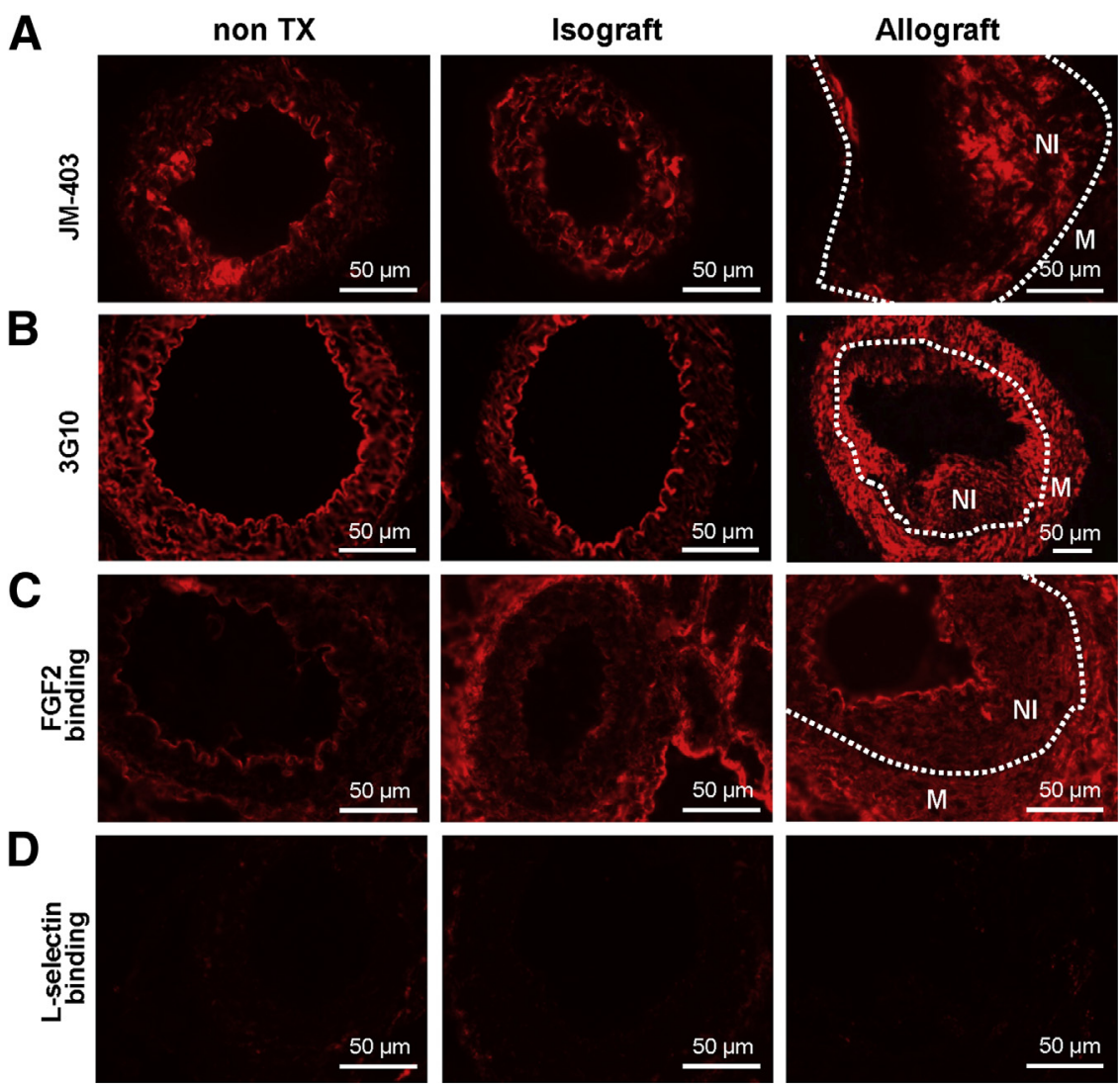

heparitinase cleavage of HS, was absent in the glomerular basement membranes of control kidneys, became weakly expressed in the isografted kidneys, and was strongly upregulated in glomerular basement membranes and sclerotic areas in the allografts (Figure 3B). The 3G10 staining showed colocalization with perlecan (Figure 4), which might suggest expression by perlecan. FGF-2 binding was completely HS-dependent, as evidenced by control experiments on sections pretreated with heparitinase, in which all binding of FGF-2 was lost (data not shown). In glomeruli of control kidneys and isografts, FGF-2 binding to HS was absent or only weakly present, but increased binding of FGF-2 was observed in allografts (Figure 3C). Although L-selectin clearly bound to HS in Bowman's capsule and to $\mathrm{HS}$ in a number of tubular basement membranes, no glomerular staining was observed in control, isografted, or allografted kidneys (Figure 3D).

In the arterial mediae of control and isografted kidneys, moderate staining for anti-HS mAbs JM-403 and 3G10 was observed (Figure 5, A and B); however, binding capacity for FGF2 and L-selectin was absent (Figure 5, C and D). In contrast to staining in the mediae of control and isografted kidneys, JM-403 staining was lost in allograft mediae, but 3 G10 staining was increased (Figure 5, A and B). Moreover, FGF2 binding capacity was increased in the allograft mediae (Figure 5C). In the neointimae, strong binding of anti-HS mAbs JM-403 and 3G10 was observed, as well as binding of FGF2 (Figure 5, A-C). L-selectin did not bind to arterial HS of control, isografted, or allografted kidneys

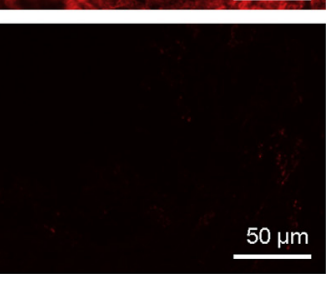

Figure 5 Modification of arterial HS structure in allografts with CTD. Medial and neointimal expression of HS was evaluated by two anti-HS mAbs, JM-403 (A) and 3G10 (B), as well as by binding of FGF2 (C) and L-selectin (D). In the media of allografted kidneys, JM-403 staining is largely lost, whereas in the neointima it is clearly expressed (A). Both the media and neointima of the allografts express the $3 \mathrm{G} 10$ HS epitope (B). The media and neointima bind FGF2 (C), but not L-selectin (D). Negative controls are shown in Figure 3. Although L-selectin does not bind to arteries, HS-dependent binding was seen in Bowman's capsule and tubular basement membranes (Figure 3). Scale bar $=50 \mu \mathrm{m}$.

(Figure 5D), despite recognition of HS isoforms in other renal structures, such as Bowman's capsule and tubular basement membranes (Figure 3).

Taken together, these findings indicate changes in glomerular and arterial HS composition in allografts with CTD, loss of JM-403 staining, and increased FGF2 binding capacity, without L-selectin binding capacity. Based on the epitope requirements (Table 2), this can be explained by increased HS sulfation (most likely $\mathrm{N}$-sulfation and 2-O-sulfation, but not 6-O-sulfation).

\section{FGF2 Binding to Perlecan HS Requires Specific Sulfation Motifs}

In an ELISA approach, we evaluated the interaction of recFGF2 with immobilized perlecan and studied competition of FGF2-perlecan interaction by fluid phase glycosaminoglycans. As expected, heparin dose-dependently inhibited the interaction of FGF2 with the HS side chains of perlecan. N-desulfation of heparin largely reduced the inhibitory capacity of heparin, and O-desulfation completely prevented interaction with FGF2 (Figure 6A). Dosedependent inhibition was also achieved by HS isolated from bovine kidney, although to a lesser extent than heparin. $\mathrm{N}$-deacetylation followed by $\mathrm{N}$-resulfation of $\mathrm{HS}$ from bovine kidney clearly increased the inhibitory capacity (Figure 6B), whereas low-sulfated HS from mouse EHS tumor or from human aorta inhibited FGF2-perlecan interaction only to some extent or not at all. These findings 

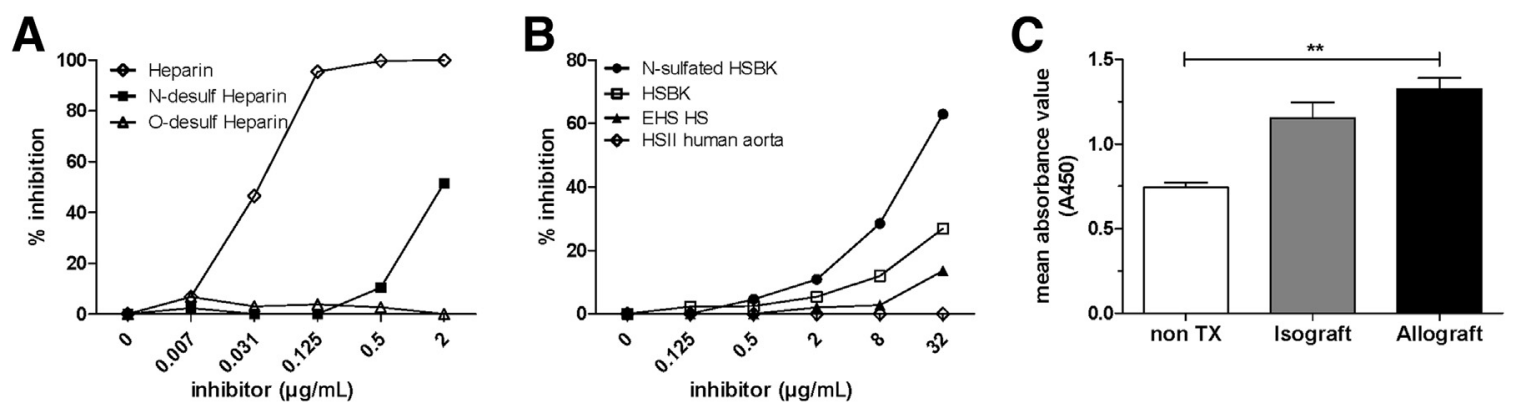

Figure 6 FGF2 binding to perlecan. A and B: Inhibition of the binding of recFGF2 to immobilized perlecan by fluid-phase GAGs was measured by ELISA. Heparin dose-dependently inhibits FGF2 from binding to immobilized perlecan. $\mathrm{N}$-desulfated heparin largely loses inhibitory capacity and 0-desulfated heparin completely loses binding to FGF2 (A). HS from bovine kidney dose-dependently inhibits FGF2 binding to perlecan (B). N-sulfated HS from bovine kidney increases binding capacity to FGF2. In contrast, two less sulfated HS preparations (from EHS sarcoma and human aorta) bind poorly with FGF2. C: FGF2 binding to antibody-captured perlecan from renal lysates indicates increased FGF2 binding to perlecan from allografted kidneys, compared with perlecan from control kidneys. Data are expressed as means \pm SEM (C). ${ }^{* *} P<0.01$.

show that the degree of sulfation of HS and heparin polysaccharides determines interaction with FGF2.

Next, we evaluated the FGF2 binding capacity of perlecan selectively adsorbed from renal lysates by immobilized anti-rat perlecan $\mathrm{mAb}$ in ELISA plates. Perlecan from allografted kidneys bound substantially more FGF2, compared with perlecan from control kidneys $(P<0.01)$
(Figure 6C), because of increased sulfation and/or content of perlecan from allografted kidneys.

\section{Endogenous Expression of FGF2 in Allografts with CTD}

We next evaluated endogenous expression levels of FGF2. In control kidneys, we observed only weak segmental FGF2
A

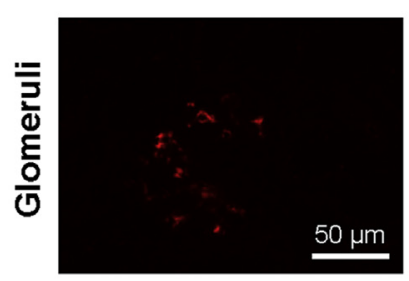

B

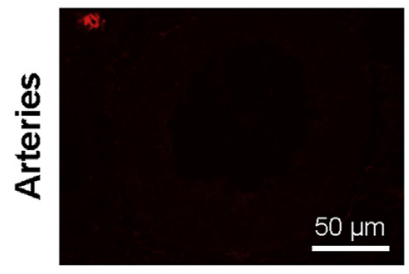

C

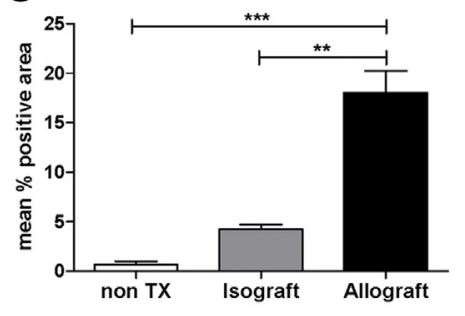

Isograft

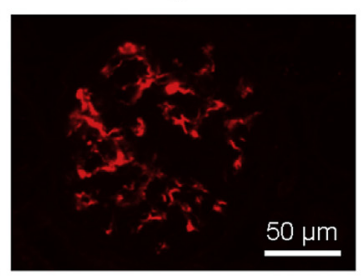

Isograft
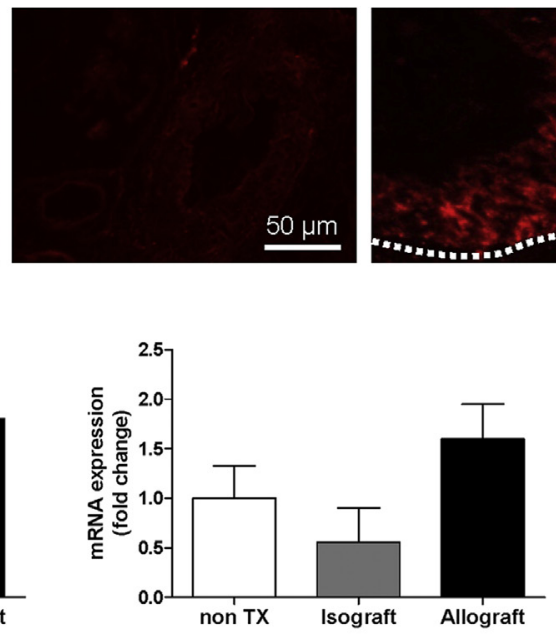

Allograft

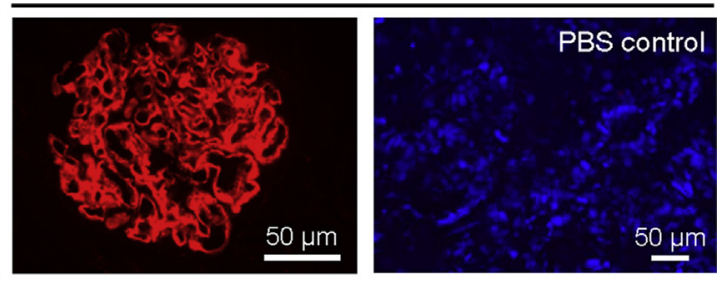

Allograft

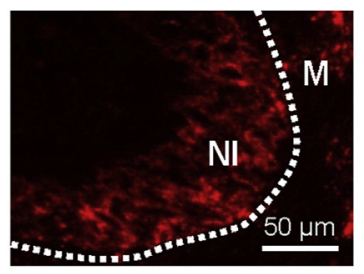

D

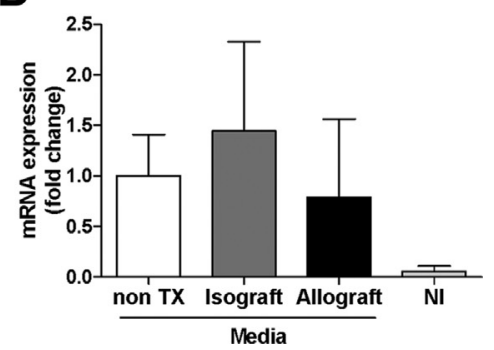

Figure 7 Glomerular and arterial expression of FGF2 in allografts with CTD. Endogenous expression of FGF2 is evaluated by immunofluorescence (A and B) and RT-qPCR (C and D). In allografts with CTD, a strong expression of FGF2 is exclusively found in glomeruli (A) and neointimae (B). In glomeruli of isografts, a slight increase in FGF2 expression is found (A). The internal elastic lamina is marked by a dotted line. C: Quantification of glomerular FGF2 expression by digital image analysis shows significant increase in allografted kidneys (left). RT-qPCR analysis revealed no increase in FGF2 expression in the glomeruli in allografts, compared with isografts and nontransplanted control tissue (right). D: Neointimal expression of FGF2. The blank (PBS control) was stained without incubation by anti-FGF2 mAb, in the presence of DAPI to show the nuclei of renal allograft tissue. Data are expressed as means \pm SEM. ${ }^{* * P}<0.01,{ }^{* *} P<$ 0.001. $n=5$ per group. Scale bar $=50 \mu \mathrm{m}$. 
A
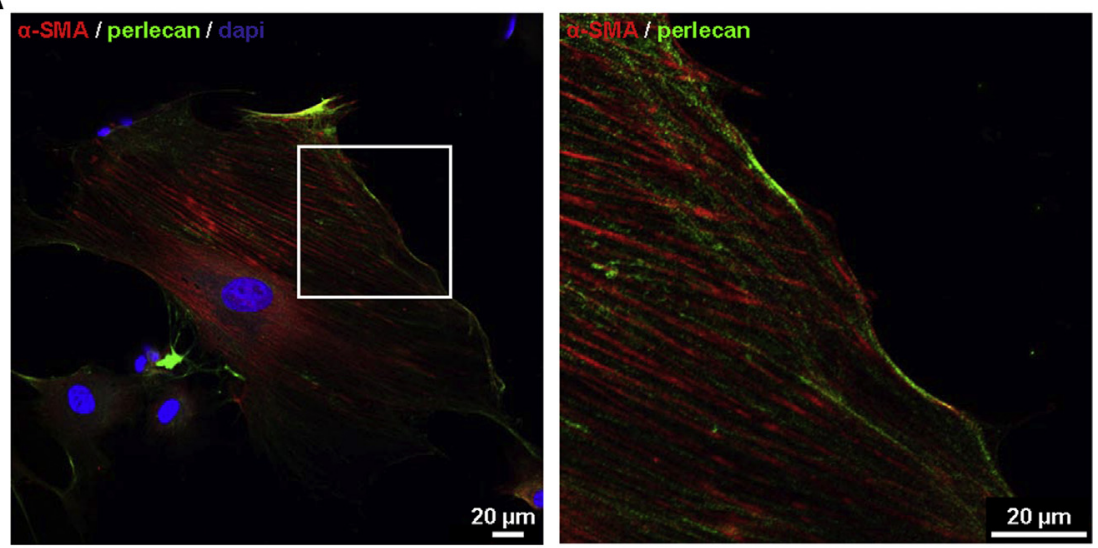

B
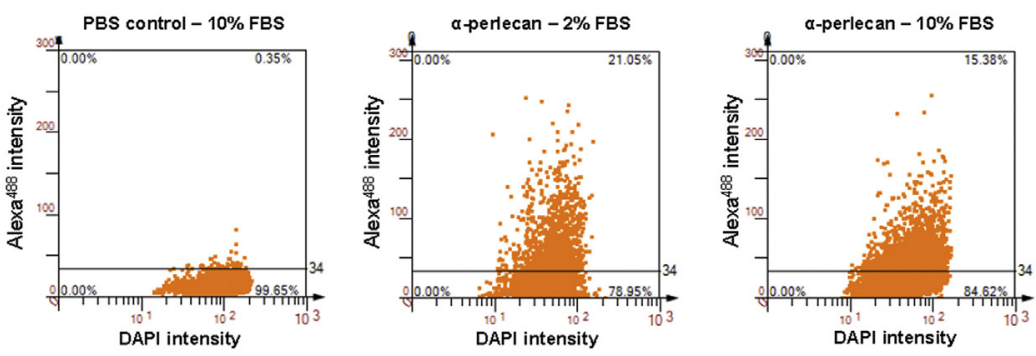

C
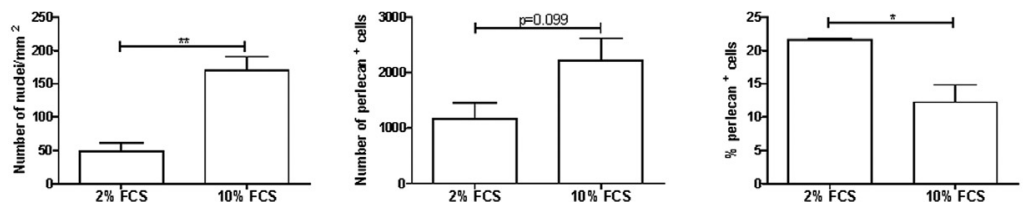

Figure 8 Proliferating mesangial cells express perlecan on their cell membranes. A: Proliferating rat mesangial cells were double-stained for $\alpha$-smooth muscle actin and perlecan to show perlecan expression on the cell membranes of proliferating mesangial cells. The boxed region is shown at higher magnification in the right image. The image is a compressed confocal Z-scan of $2.63 \mu \mathrm{m}$ consisting of seven stacks. B and C: Comparison of cell numbers (based on DAPI staining) and perlecan expression (Alexa Fluor 488) in resting (2\% FCS) and proliferating $(10 \% \mathrm{FCS})$ mesangial cell cultures, presented as tissue fluorescence-activated cell-sorting scatter plots (B) and as quantification of the data (C). $10 \%$ FCS induces significant proliferation of the mesangial cells, with an increase in the absolute numbers of perlecan-positive mesangial cells. Relatively, the percentage of perlecan-positive cells is decreased after culturing with 10\% FCS, compared with $2 \%$ FCS. Data are expressed as means \pm SEM (C). ${ }^{*} P<0.05,{ }^{* *} P<0.01$. Scale bar $=20 \mu \mathrm{m}$.

expression in the glomeruli and the tubulointerstitium (Figure 7A). Arteries were devoid of any FGF2 expression (Figure 7B). In isografts, a slight up-regulation of FGF2 expression in glomeruli was observed, with the arteries remaining devoid of any FGF2 expression (Figure 7, A and B). In the allografts, however, FGF2 was strongly and homogeneously accumulated throughout the glomeruli and was present mainly in the glomerular capillary wall and mesangium (Figure 7A). Quantification of FGF2 staining by digital image analysis revealed strong increased FGF2 expression in the glomeruli of allografted kidneys, compared with isografted kidneys $(P<0.02)$ and nontransplanted control kidneys $(P<0.01)$ (Figure $7 \mathrm{C})$. PCR analysis revealed no significant differences in glomerular FGF2 expression, although a tendency toward higher expression was observed in the allografts (Figure 7C). We suggest that this discrepancy may be explained by plasmaderived FGF2 being trapped in the glomeruli by perlecan endowed with HS chains able to bind FGF2 (Figure 3) during ultrafiltration; this would cause local accumulation of FGF2 protein in the absence of any increase in mRNA expression. In the neointimae of allografts, increased FGF2 expression was observed at the protein level, but not at the mRNA level (Figure 7, B and D).

\section{Involvement of Proteoglycans in FGF2-Driven} Proliferation of Mesangial Cells

To explore a possible mechanistic relation between glomerular HSPGs and FGF2 in greater detail, we conducted in vitro experiments. Primary rat mesangial cells were cultured and stained for $\alpha$-smooth muscle actin and perlecan. A confocal image of cultured mesangial cells stained for $\alpha$-smooth muscle actin (activation marker of mesangial cells) and perlecan reveals that activated, proliferating mesangial cells express perlecan, and magnification of a Z-stack compilation clearly demonstrated the extracellular presence of perlecan positioned on the cell membrane (Figure 8A). We next compared perlecan expression in resting (2\% FCS culture conditions) and proliferating (10\% FCS culture conditions) mesangial cells. Quantitative tissue fluorescence-activated cell sorting analysis revealed a proliferative response for mesangial cells cultured with $10 \%$ FCS (Figure 8, B and C). The absolute number of perlecanpositive mesangial cells increased under $10 \%$ FCS conditions; the percentage, however, decreased.

To demonstrate proliferation of mesangial cells in allografted kidneys in vivo, double-staining was performed using mesangial cell marker Thy-1 (alias CD90) with Ki-67 as 
A

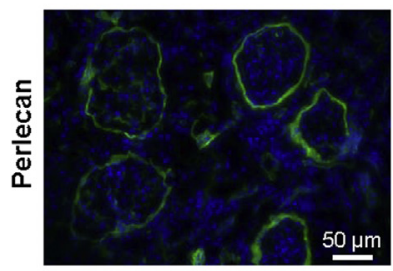

B
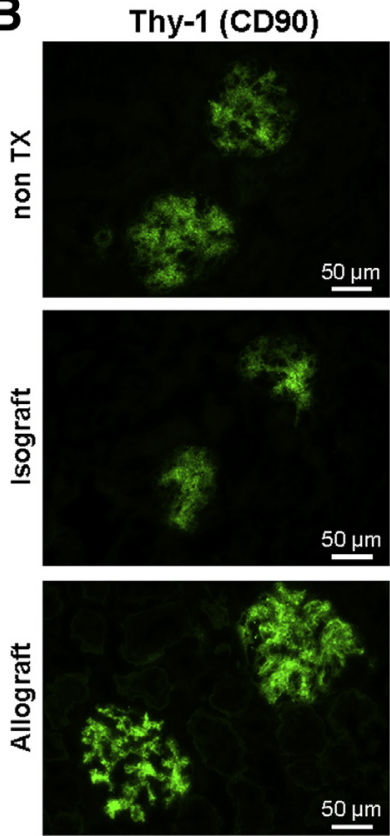

Isograft

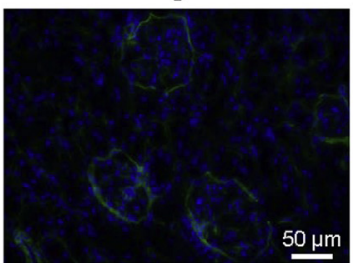

Ki67
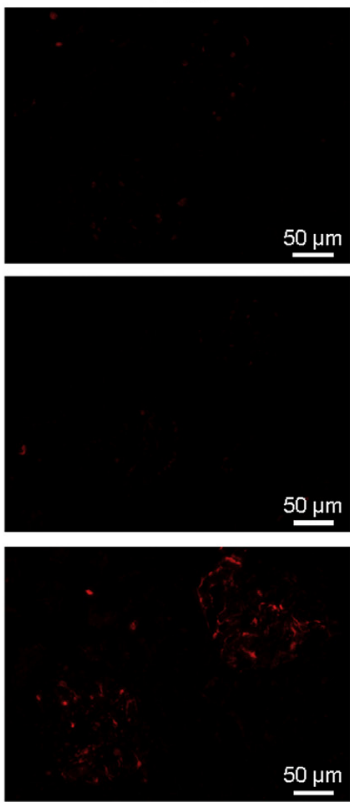
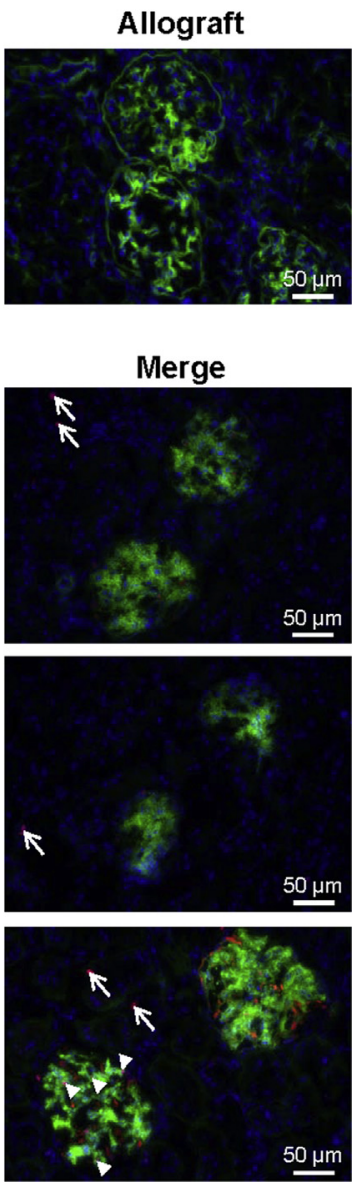

Figure 9 Proliferation of perlecan-positive mesangial cells in allografted kidneys. A: Kidneys from nontransplanted controls, isografted, and allografted rats were stained for perlecan. Strong induction of perlecan is seen in the glomeruli of the allografts. B: In the same kidneys, doublestaining was performed using $0 \times 7$ (Thy- 1 ; alias CD90) as a rat mesangial phenotypic marker and $\mathrm{Ki}-67$ as a proliferation marker. Staining reveals presence of Ki-67-positive nuclei (arrowheads) in the Thy-1-positive mesangial areas within the glomeruli of allografted kidneys but not of isografted or nontransplant control kidneys. Ki-67positive proliferating interstitial cells are indicated by arrows. Scale bar $=50 \mu \mathrm{m}$. a proliferation marker. In allografted kidneys, but not in isografted and nontransplant control kidneys, the number of Ki-67-positive nuclei was increased in Thy-1-positive mesangial areas within the glomeruli (Figure 9B). Perlecan expression in the same kidneys demonstrated that mesangial proliferation associates with increased perlecan in the same allografts (Figure 9A).

We observed a dose-dependent increase in proliferation of mesangial cells in response to FGF2 (Figure 10A). The FGF2-induced proliferative response was blunted by the addition of exogenous competitive bovine kidney-derived HS (Figure 10B), because of reduced binding of FGF2 to the cells, as demonstrated by reduced binding of ${ }^{125}$ I-labeled FGF2 to the mesangial cells (data not shown). To verify that proliferation of the mesangial cells is dependent on FGF2 binding by endogenous HS, cells were cultured in the presence of chlorate, which inhibited the sulfation of the side chains of the proteoglycans in a dose-dependent manner (Figure 10C). Chlorate prevented the FGF2-induced proliferative response of mesangial cells, which appeared to be dependent on the duration of FGF2 stimulation (Figure 10D). These results indicate that the proliferation of mesangial cells is dependent on FGF2 binding to endogenous HS proteoglycans such as perlecan. The interaction between FGF2 and HS proteoglycans on mesangial cells may therefore play a key role in the development of FGS.

\section{Discussion}

Here we have provided evidence that matrix HSPGs such as perlecan serve as functional docking platforms for FGF2 and contribute to glomerular and arterial tissue remodeling in experimental CTD. The concept of extracellular regulation of growth factors by proteoglycans has been studied predominantly in tissue remodeling related to embryonic development, ${ }^{14,15}$ ontogenesis, ${ }^{16,17}$ and angiogenesis. ${ }^{18,19}$ The present results indicate that HSPGs modulate growth factors in the renal transplant setting as well. This important finding opens the possibility of targeting proteoglycans by therapeutic intervention to ameliorate the development of CTD.

Development of CTD in renal allografts is the result of tissue remodeling that affects all functional and structural compartments of the kidney, including the tubulointerstitium (tubular atrophy and interstitial fibrosis), glomeruli (focal glomerulosclerosis), and larger arteries (neointima formation). ${ }^{2-4}$ We previously demonstrated compositional changes of the HSPGs collagen type XVIII, perlecan, and agrin in tissue remodeling in experimental CTD by immunofluorescence. ${ }^{5}$ We investigated functional aspects of proteoglycans in experimental CTD and in renal cell culture assays. Depending on their highly variable composition, HS carbohydrate side chains can bind a variety of ligands, 

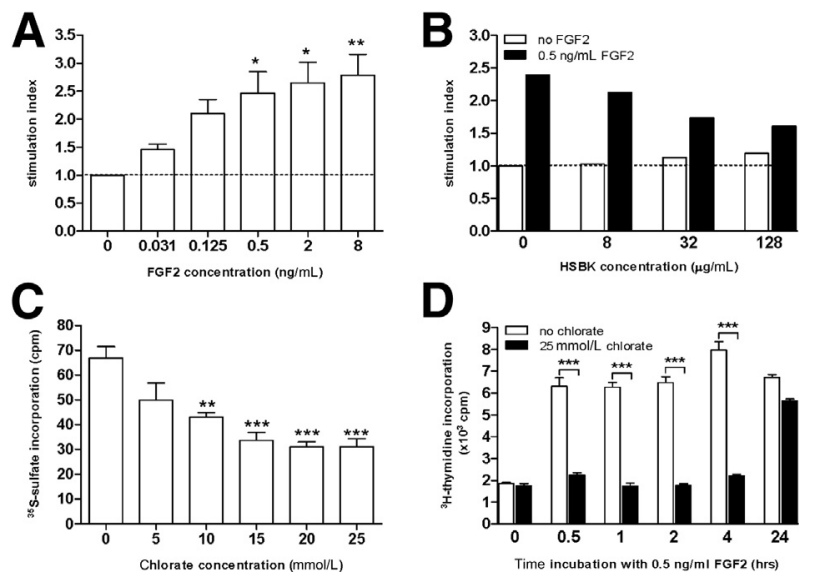

Figure 10 FGF2-induced proliferation of mesangial cells depends on sulfated proteoglycans. A: Rat primary mesangial cells were stimulated with various concentrations of FGF2 for 24 hours. Proliferation was measured by $\left[{ }^{3} \mathrm{H}\right]$ thymidine incorporation. The stimulation index is calculated as fold increase in proliferation + FGF2/proliferation - FGF2. B: Exogenous HS from bovine kidney (HSBK) hampers FGF2-induced mesangial cell proliferation in a dose-dependent manner. C: Proteoglycan sulfation of mesangial cells is reduced by chlorate in a dose-dependent manner. D: Chlorate impairs FGF2-induced proliferation of mesangial cells, which is dependent on the duration of FGF2 stimulation. Data are expressed as means \pm SEM $\left(\mathbf{A}, \mathbf{C}\right.$, and D). $n=4(\mathbf{A}) ; n=2(\mathbf{B}) .{ }^{*} P<0.05,{ }^{*} P P<0.01$, and ${ }^{* * *} P<$ 0.001 versus control.

including FGF2, ${ }^{12}$ which is known to be involved in renal tissue remodeling. In experimental allografts with CTD, a massive increase in matrix HSPG-mediated binding capacity for FGF2 was observed. Enhanced binding capacity for FGF2 was the result of increased HSPG expression and modified HS sulfation. Result indicate increased $\mathrm{N}$-sulfation and 2-O-sulfation leading to loss of JM-403 staining ${ }^{28}$ and increased FGF2 binding, ${ }^{32}$ but without an increase in 6-O-sulfation, because 6-O-sulfate-dependent L-selectin binding $^{33}$ was not induced in glomerular and neointimal HS. This finding suggests that on allotransplantation the kidney mobilizes matrix HSPGs to become docking molecules for heparin-binding growth factors such as FGF2. In native kidney diseases, Morita et $\mathrm{al}^{34}$ found increased FGF2 binding in fibrotic areas.

The laser microdissection approach on isolated glomeruli, arterial mediae, and neointimae revealed spatial differences in HSPG expression that were not seen (and would have been missed) in whole-kidney homogenates. We observed increased glomerular and neointimal perlecan expression in allografted kidneys. This regional HSPG regulation opposes the uniform induction of profibrotic pathways (ColI $\alpha 1$, ColIV $\alpha 1$, and TGF- $\beta 1$ ) seen in glomeruli, mediae, and whole-kidney tissue, which probably reflects a more generalized response on allotransplantation. Like perlecan, FGF2 also showed a restricted glomerular and neointimal expression pattern. These findings indicate that the perlecan-FGF2 axis is not relevant for the interstitial fibrosis seen in this rat CTD model. Apparently, tubulointerstitial fibrosis is driven by other (proteoglycan-independent) growth factors, such as TGF- $\beta 1$. The marked expression of FGF2 and perlecan observed in glomeruli of allografted kidneys suggests a role in the proliferative response of mesangial cells, which was substantiated by the finding of Ki-67-positive nuclei within Thy-1-positive mesangial cells in allografted kidneys. In other settings, perlecan and FGF2 have been shown to induce proliferation in a number of cell types, including chondrocytes, neural stem cells, retinal pigmental epithelial cells, and vascular smooth muscle cells.

In the kidney, FGF2 has been shown to be proliferative for fibroblasts and mesangial cells. ${ }^{38,39}$ In vitro studies showed that the expression of cell-surface HSPG was a prerequisite for the proliferation of renal fibroblasts in response to FGF2. ${ }^{38}$ We therefore examined the functional role of the FGF2-proteoglycan interaction in mesangial cell proliferation in vitro. FGF2 was shown to induce mesangial cell proliferation in a dose- and sulfation-dependent manner, which favors a role for the FGF2-proteoglycan interaction in mesangial cell proliferation. The exact mechanism by which HSPGs enhance FGF2-induced mesangial cell proliferation is unknown. We propose that binding of FGF2 to mesangial (matrix) HSPGs such as perlecan facilitates the interaction between FGF2 and its surface receptor on mesangial cells, as has been demonstrated for other cell types. ${ }^{35-40}$ This is supported by our observation that removing the FGF2 binding capacity of proteoglycans on mesangial cells by chlorate treatment resulted in a significant delay in (but not complete abrogation of) FGF2induced proliferation. In a separate series of experiments, we excluded a direct effect of perlecan on a fibrogenic response (RT-qPCR for ColI $\alpha \mathrm{I}$, ColIV $\alpha \mathrm{I}$, and TGF- $\beta 1$ ) by the mesangial cells (data not shown).

Based on our data, HSPGs could hold promise as therapeutic targets to limit CTD, especially focused on the potential of inhibiting growth factor signaling. Addressing the possibility that exogenous glycosaminoglycans may hamper growth factor responses, heparin and heparinoids have been shown to reduce progressive renal failure in experimental renal diseases, including renal transplantation. ${ }^{41-43}$ Increasingly, research interest is focusing on the possibility of producing small HS mimetics, which may more specifically target a particular component of HS and heparin bioactivity. ${ }^{44}$ The use of HSPGs as targets (for example, using antibodies that recognize and thereby block specific HS motifs or domains) may also have clinical potential. This strategy has been exemplified in vitro by the demonstration that 6-O-sulfate-specific anti-HS antibodies produced in a phage-display library can inhibit leukocyte rolling and firm adhesion to glomerular endothelial cells, whereas anti-HS antibodies with different specificities do not. ${ }^{45}$ Mutant growth factors that are rendered incapable of glycosaminoglycan binding could be used to specifically inhibit cell survival and/or proliferation. In addition, small inactive growth factor fragments could be generated that block the HSPGbinding sites of their in vivo active counterparts. Thus, there are various options for the use of HSPGs in therapeutic strategies, but further proof of efficacy in vivo needs to be provided. 


\section{Acknowledgments}

We thank Peter Zwiers, Marian Bulthuis, André Zandvoort, and Annemieke Smit-van Oosten for their excellent technical assistance.

\section{Supplemental Data}

Supplemental material for this article can be found at http://dx.doi.org/10.1016/j.ajpath.2013.07.030.

\section{References}

1. Kouwenhoven EA, IJzermans JN, de Bruin RW: Etiology and pathophysiology of chronic transplant dysfunction. Transpl Int 2000, 13: 385-401

2. Chapman JR, O'Connell PJ, Nankivell BJ: Chronic renal allograft dysfunction. J Am Soc Nephrol 2005, 16:3015-3026

3. Solez K, Colvin RB, Racusen LC, Haas M, Sis B, Mengel M, Halloran PF, Baldwin W, Banfi G, Collins AB, Cosio F, David DS, Drachenberg C, Einecke G, Fogo AB, Gibson IW, Glotz D, Iskandar SS, Kraus E, Lerut E, Mannon RB, Mihatsch M, Nankivell BJ, Nickeleit V, Papadimitriou JC, Randhawa P, Regele H, Renaudin K, Roberts I, Seron D, Smith RN, Valente M: Banff 07 classification of renal allograft pathology: updates and future directions. Am J Transplant 2008, 8:753-760

4. Solez K, Colvin RB, Racusen LC, Sis B, Halloran PF, Birk PE, Campbell PM, Cascalho M, Collins AB, Demetris AJ, Drachenberg CB, Gibson IW, Grimm PC, Haas M, Lerut E, Liapis H, Mannon RB, Marcus PB, Mengel M, Mihatsch MJ, Nankivell BJ, Nickeleit V, Papadimitriou JC, Platt JL, Randhawa P, Roberts I, Salinas-Madriga L, Salomon DR, Seron D, Sheaff M, Weening JJ: Banff '05 Meeting Report: differential diagnosis of chronic allograft injury and elimination of chronic allograft nephropathy ('CAN'). Am J Transplant 2007, 7:518-526

5. Rienstra H, Katta K, Celie JW, van Goor H, Navis G, van den Born J, Hillebrands JL: Differential expression of proteoglycans in tissue remodeling and lymphangiogenesis after experimental renal transplantation in rats. PLoS One 2010, 5:e9095

6. Celie JW, Reijmers RM, Slot EM, Beelen RH, Spaargaren M, Ter Wee PM, Florquin S, van den Born J: Tubulointerstitial heparan sulfate proteoglycan changes in human renal diseases correlate with leukocyte influx and proteinuria. Am J Physiol Renal Physiol 2008, 294:F253-F263

7. Celie JW, Rutjes NW, Keuning ED, Soininen R, Heljasvaara R, Pihlajaniemi T, Dräger AM, Zweegman S, Kessler FL, Beelen RH, Florquin S, Aten J, van den Born J: Subendothelial heparan sulfate proteoglycans become major L-selectin and monocyte chemoattractant protein-1 ligands upon renal ischemia/reperfusion. Am J Pathol 2007, 170:1865-1878

8. Zaferani A, Vives RR, van der Pol P, Hakvoort JJ, Navis GJ, van Goor H, Daha MR, Lortat-Jacob H, Seelen MA, van den Born J: Identification of tubular heparan sulfate as a docking platform for the alternative complement component properdin in proteinuric renal disease. J Biol Chem 2011, 286:5359-5367

9. Whitelock JM, Melrose J, Iozzo RV: Diverse cell signaling events modulated by perlecan. Biochemistry 2008, 47:11174-11183

10. Iozzo RV, Sanderson RD: Proteoglycans in cancer biology, tumour environment and angiogenesis. J Cell Mol Med 2011, 15:1013-1031

11. Lortat-Jacob H: The molecular basis and functional implications of chemokine interactions with heparan sulphate. Curr Opin Struct Biol 2009, 19:543-548

12. Guimond S, Maccarana M, Olwin BB, Lindahl U, Rapraeger AC: Activating and inhibitory heparin sequences for FGF-2 (basic FGF).
Distinct requirements for FGF-1, FGF-2, and FGF-4. J Biol Chem 1993, 268:23906-23914

13. Gallagher JT, Turnbull JE: Heparan sulphate in the binding and activation of basic fibroblast growth factor. Glycobiology 1992, 2:523-528

14. Schwabiuk M, Coudiere L, Merz DC: SDN-1/syndecan regulates growth factor signaling in distal tip cell migrations in C. elegans. Dev Biol 2009, 334:235-242

15. Olivares GH, Carrasco H, Aroca F, Carvallo L, Segovia F, Larraín J: Syndecan-1 regulates BMP signaling and dorso-ventral patterning of the ectoderm during early Xenopus development. Dev Biol 2009, 329: 338-349

16. Menashe I, Maeder D, Garcia-Closas M, Figueroa JD, Bhattacharjee S, Rotunno M, Kraft P, Hunter DJ, Chanock SJ, Rosenberg PS, Chatterjee N: Pathway analysis of breast cancer genome-wide association study highlights three pathways and one canonical signaling cascade. Cancer Res 2010, 70:4453-4459

17. Götte M, Kersting C, Radke I, Kiesel L, Wülfing P: An expression signature of syndecan-1 (CD138), E-cadherin and c-met is associated with factors of angiogenesis and lymphangiogenesis in ductal breast carcinoma in situ. Breast Cancer Res 2007, 9:R8

18. Iozzo RV, Zoeller JJ, Nyström A: Basement membrane proteoglycans: modulators par excellence of cancer growth and angiogenesis. Mol Cells 2009, 27:503-513

19. Aviezer D, Hecht D, Safran M, Eisinger M, David G, Yayon A: Perlecan, basal lamina proteoglycan, promotes basic fibroblast growth factor-receptor binding, mitogenesis, and angiogenesis. Cell 1994, 79: $1005-1013$

20. Nakamura T, Ebihara I, Fukui M, Osada S, Nagaoka I, Horikoshi S, Tomino Y, Koide H: Messenger RNA expression for growth factors in glomeruli from focal glomerular sclerosis. Clin Immunol Immunopathol 1993, 66:33-42

21. Floege J, Kriz W, Schulze M, Susani M, Kerjaschki D, Mooney A, Couser WG, Koch KM: Basic fibroblast growth factor augments podocyte injury and induces glomerulosclerosis in rats with experimental membranous nephropathy. J Clin Invest 1995, 96:2809-2819

22. Kriz W, Hähnel B, Rösener S, Elger M: Long-term treatment of rats with FGF-2 results in focal segmental glomerulosclerosis. Kidney Int 1995, 48:1435-1450

23. Chan J, Prado-Lourenco L, Khachigian LM, Bennett MR, Di Bartolo BA, Kavurma MM: TRAIL promotes VSMC proliferation and neointima formation in a FGF-2-, Sp1 phosphorylation-, and NFkappaB-dependent manner. Circ Res 2010, 106:1061-1071

24. Camozzi M, Zacchigna S, Rusnati M, Coltrini D, Ramirez-Correa G, Bottazzi B, Mantovani A, Giacca M, Presta M: Pentraxin 3 inhibits fibroblast growth factor 2-dependent activation of smooth muscle cells in vitro and neointima formation in vivo. Arterioscler Thromb Vasc Biol 2005, 25:1837-1842

25. Rienstra H, Boersema M, Onuta G, Boer MW, Zandvoort A, van Riezen M, Rozing J, van Goor H, Navis GJ, Popa ER, Hillebrands JL: Donor and recipient origin of mesenchymal and endothelial cells in chronic renal allograft remodeling. Am J Transplant 2009, 9:463-472

26. Boersema M, Katta K, Rienstra H, Molema G, Nguyen TQ, Goldschmeding R, Navis G, van den Born J, Popa ER, Hillebrands JL: Local medial microenvironment directs phenotypic modulation of smooth muscle cells after experimental renal transplantation. Am J Transplant 2012, 12:1429-1440

27. Asgeirsdottir SA, Werner N, Harms G, Van Den Berg A, Molema G: Analysis of in vivo endothelial cell activation applying RT-PCR following endothelial cell isolation by laser dissection microscopy. Ann N Y Acad Sci 2002, 973:586-589

28. van den Born J, Gunnarsson K, Bakker MA, Kjellén L, KuscheGullberg M, Maccarana M, Berden JH, Lindahl U: Presence of Nunsubstituted glucosamine units in native heparan sulfate revealed by a monoclonal antibody. J Biol Chem 1995, 270:31303-31309

29. David G, Bai XM, Van der Schueren B, Cassiman JJ, Van den Berghe H: Developmental changes in heparan sulfate expression: in situ detection with mAbs. J Cell Biol 1992, 119:961-975 
30. Celie JW, Beelen RH, van den Born J: Effect of fixation protocols on in situ detection of L-selectin ligands. J Immunol Methods 2005, 298: 155-159

31. Iverius PH: Coupling of glycosaminoglycans to agarose beads (Sepharose 4B). Biochem J 1971, 124:677-683

32. Maccarana M, Casu B, Lindahl U: Minimal sequence in heparin/ heparan sulfate required for binding of basic fibroblast growth factor [Erratum appeared in J Biol Chem 1994, 269:3903]. J Biol Chem 1993, 268:23898-23905

33. Wang L, Brown JR, Varki A, Esko JD: Heparin's anti-inflammatory effects require glucosamine 6-O-sulfation and are mediated by blockade of L- and P-selectins. J Clin Invest 2002, 110:127-136

34. Morita H, Shinzato T, David G, Mizutani A, Habuchi H, Fujita Y, Ito M, Asai J, Maeda K, Kimata K: Basic fibroblast growth factorbinding domain of heparan sulfate in the human glomerulosclerosis and renal tubulointerstitial fibrosis. Lab Invest 1994, 71:528-535

35. Smith SM, West LA, Govindraj P, Zhang X, Ornitz DM, Hassell JR: Heparan and chondroitin sulfate on growth plate perlecan mediate binding and delivery of FGF-2 to FGF receptors [Erratum appeared in Matrix Biol 2007, 26:583]. Matrix Biol 2007, 26:175-184

36. Guillonneau X, Tassin J, Berrou E, Bryckaert M, Courtois Y, Mascarelli F: In vitro changes in plasma membrane heparan sulfate proteoglycans and in perlecan expression participate in the regulation of fibroblast growth factor 2 mitogenic activity. J Cell Physiol 1996, 166:170-187

37. Park Y, Rangel C, Reynolds MM, Caldwell MC, Johns M, Nayak M, Welsh CJ, McDermott S, Datta S: Drosophila perlecan modulates FGF and hedgehog signals to activate neural stem cell division. Dev Biol 2003, 253:247-257

38. Clayton A, Thomas J, Thomas GJ, Davies M, Steadman R: Cell surface heparan sulfate proteoglycans control the response of renal interstitial fibroblasts to fibroblast growth factor-2. Kidney Int 2001, 59:2084-2094

39. Francki A, Uciechowski P, Floege J, von der Ohe J, Resch K Radeke HH: Autocrine growth regulation of human glomerular mesangial cells is primarily mediated by basic fibroblast growth factor. Am J Pathol 1995, 147:1372-1382

40. Strutz F: The role of FGF-2 in renal fibrogenesis. Front Biosci (Schol Ed) 2009, 1:125-131

41. van Bruggen MC, Walgreen B, Rijke TP, Corsius MJ, Assmann KJ, Smeenk RJ, van Dedem GW, Kramers K, Berden JH: Heparin and heparinoids prevent the binding of immune complexes containing nucleosomal antigens to the GBM and delay nephritis in MRL/lpr mice. Kidney Int 1996, 50:1555-1564

42. Gottmann U, Mueller-Falcke A, Schnuelle P, Birck R, Nickeleit V, van der Woude FJ, Yard BA, Braun C: Influence of hypersulfated and low molecular weight heparins on ischemia/reperfusion: injury and allograft rejection in rat kidneys. Transpl Int 2007, 20: $542-549$

43. Ceol M, Gambaro G, Sauer U, Baggio B, Anglani F, Forino M, Facchin S, Bordin L, Weigert C, Nerlich A, Schleicher ED: Glycosaminoglycan therapy prevents TGF-betal overexpression and pathologic changes in renal tissue of long-term diabetic rats. J Am Soc Nephrol 2000, 11:2324-2336

44. Ashikari-Hada S, Habuchi H, Sugaya N, Kobayashi T, Kimata K: Specific inhibition of FGF-2 signaling with 2-O-sulfated octasaccharides of heparan sulfate. Glycobiology 2009, 19:644-654

45. Rops AL, van den Hoven MJ, Baselmans MM, Lensen JF, Wijnhoven TJ, van den Heuvel LP, van Kuppevelt TH, Berden JH, van der Vlag J: Heparan sulfate domains on cultured activated glomerular endothelial cells mediate leukocyte trafficking. Kidney Int 2008, 73: $52-62$ 